\title{
MODELING CREATIVITY AND COMMUNICATION
}

\author{
JUSTIN SULIK ${ }^{1}$ \\ *justin.sulik@gmail.com \\ ${ }^{1}$ Department of Psychology, Royal Holloway, University of London
}

Usually, humans use shared language to communicate successfully and efficiently. But people can also communicate - with considerably more effort and less predictable success - by creatively inventing novel signals. Modern humans need to do this comparatively rarely: when playing guessing games, or when interacting with people with whom they do not share a language. However, our prelinguistic ancestors had no shared words, so the creative generation of novel signals was one plausible route to conventional language.

This raises a cognitive puzzle. On one hand, the inferential mechanisms that underlie the creative generation of novel signals are computationally complex (Levinson, 1995, 2006; Stolk et al., 2013), and while any neurotypical human can learn language given the right input, performance at creative signaling is a great deal less uniform (Sulik \& Lupyan, in prep.). On the other hand, the creative generation of novel signals was evolutionarily prior to the emergence of language.

Thus, creativity is a cognitively expensive bump in the road to language. The puzzle is how we got over that bump. Generating or interpreting a novel signal requires extensive investment of cognitive resources, and this investment does not predictably lead to communicative success. In this work I ask, 'in what situations do the computationally expensive mechanisms of creativity boost communicative success?' This question is tackled here with agent-based simulations of creativity and communication. This allows explicit manipulation of relevant features, such as agents' mental representations, communicative strategies, and context.

First, I review psychological accounts of the cognitive mechanisms that underlie creativity. For instance, Mednick (1962) characterizes creativity as involving a relatively flat associative hierarchy in semantic knowledge. Jung-Beeman (2005) argues that creative problem solving involves coarse coding (weak, broad spread of activation) as opposed to fine coding (strong, focused spread of activation). Other accounts emphasize the small-world network properties of creativity (Schilling, 2005; Kenett, Anaki, \& Faust, 2014) or differences in executive control (Benedek et al., 2017). Some of these mechanisms are demonstrably related to novelty in communication (Mashal, Faust, Hendler, \& Jung-Beeman, 2007).

Second, I introduce the model. Each agent is a graph with weighted, directed 
edges (modeling a semantic network, with concepts as nodes and their associative relationships as edges). Agents interact repeatedly. In each interaction, one concept is chosen as the target to be communicated. The signaler generates a signal by sampling (probabilistically, based on edge weights) from the associates of the target in their semantic network, much like someone wanting to communicate 'moose' might gesture a semantic associate, antlers. The receiver makes a guess based on spreading activation in their semantic network when the signal nodes fire. The model explores the factors driving communicative success. There is no generational turnover or learning, though these are directions for future research.

Most of the model parameters describe the organization and structure of the agents' networks ('neural' parameters). Some of these are directly related to the above accounts of creativity (e.g. parameters governing the distribution of weights among a node's edges or the extent to which activation spreads), while others are not directly related to creativity, but do impact communicative success (e.g., how many neighbors a node has, or how similar the signaler and receiver's graphs are), and might thus be expected to interact with the creativity parameters. Other parameters ('context' parameters) describe the communicative context, such as how constrained the context is, or how complex the signal can be.

The parameters interact in complex ways to affect communication. The following are just some illustrations. It turns out that the complexity of the signal drastically alters how neural parameters drive success, and that signalers and receivers are often subject to different pressures. A flat associative hierarchy (cf. Mednick, above) makes signal generation more entropic, while a steep hierarchy decreases signaling entropy, but whether either of these boosts communicative success depends on a variety of other factors. For instance, creative, high-entropic signalers become less successful as the number of edges increases, but this effect is counteracted when the signaler's and receiver's representations become less similar, or when signals are allowed to become more complex. I introduce an $\mathrm{R}$ Shiny app to facilitate online interactive visual exploration of the parameter space.

While it may seem discouraging that no simple conclusion can be drawn about when creativity (or its opposite, rigidity) is adaptive, this result is actually informative about the evolution of language. Since the parameters interact in complex ways, then if the communicative context varies in complexity, it is adaptive to be able to flexibly employ both creative and rigid strategies. One solution is for individuals to have access to both creative and rigid computational styles; another way is to distribute the cognitive burden between individuals, so some are more creative and others more rigid. I review evidence suggesting that human social cognition makes use of both of these strategies (Jung-Beeman, 2005; Faust \& Kenett, 2014; Sulik \& Lupyan, in prep.). I propose that the first steps towards human language must have been characterized by such variation in communicative context, and an explanation of language evolution must thus encapsulate both social and cognitive divisions of labor. 


\section{References}

Benedek, M., Kenett, Y. N., Umdasch, K., Anaki, D., Faust, M., \& Neubauer, A. C. (2017). How semantic memory structure and intelligence contribute to creative thought: a network science approach. Thinking \& Reasoning, 23(2), 158-183.

Faust, M., \& Kenett, Y. N. (2014). Rigidity, chaos and integration: hemispheric interaction and individual differences in metaphor comprehension. Frontiers in Human Neuroscience, 8, 511.

Jung-Beeman, M. (2005). Bilateral brain processes for comprehending natural language. Trends in Cognitive Sciences, 9, 512-518.

Kenett, Y. N., Anaki, D., \& Faust, M. (2014). Investigating the structure of semantic networks in low and high creative individuals. Frontiers in Human Neuroscience, 8, 407.

Levinson, S. C. (1995). Interactional biases in human thinking. In Social intelligence and interaction (pp. 221-260). Cambridge University Press.

Levinson, S. C. (2006). On the human "interaction engine". In N. J. Enfield \& S. C. Levinson (Eds.), Roots of human sociality: Culture, cognition and interaction (pp. 39-69). Oxford: Berg.

Mashal, N., Faust, M., Hendler, T., \& Jung-Beeman, M. (2007). An fMRI investigation of the neural correlates underlying the processing of novel metaphoric expressions. Brain and Language, 100, 115-126.

Mednick, S. A. (1962). The associative basis of the creative process. Psychological Review, 69(3), 220-232.

Schilling, M. A. (2005). A 'small-world' network model of cognitive insight. Creativity Research Journal, 17(2-3), 121-154.

Stolk, A., Verhagen, L., Schoffelen, J.-M., Oostenveld, R., Blokpoel, M., Hagoort, P., van Rooij, I., \& Toni, I. (2013). Neural mechanisms of communicative innovation. PNAS, 110(36), 14574-14579.

Sulik, J., \& Lupyan, G. (in prep.). Individual differences in performance at a novel signaling task. 